\title{
Performance Evaluation of Layered and Cross Layered based MANETs
}

\author{
Ritika Chauhan \\ PG, Student, \\ Ambala College of \\ Engineering \& Applied Research, \\ Devsthali, Ambala (Haryana)
}

\author{
Pooja Saini \\ Sr. Assistant Professor, \\ Department of \\ Computer Science \& Engineering, \\ Ambala College of \\ Engineering \& Applied \\ Research, Devsthali, \\ Ambala (Haryana)
}

\begin{abstract}
The versatile Ad hoc Networks provides communication among wireless nodes which occurs in the wireless medium. Energy effective routing in MANET [5] is a demanding goal which should be taken under consideration. Moreover, energy effective routing is considered to be the most important design criteria for MANETs because mobile nodes will be powered by batteries with limited capacity. The architecture of ad hoc network protocol, generally based on a conventional "layered approach", has been found ineffective to deal with, energy efficient routing and breakage of links in MANET [5]. This paper proposes a Cross Layer based MANET framework to minimize the energy consumption [3] and maximize the network lifetime with comparison to conventional layered architecture. Further the different performance metrics evaluation such as PDR, energy and number of collisions based on simulated scenarios is performed for Layered and Cross Layered based MANET. Furthermore, enhancing the cross-layer communication [1, 18] among different layers, such as network layer, MAC layer and physical layer. The proposed Cross-Layer based AODV (CLAODV) would help to minimize energy consumption and to find an effectual route between the source and the destination, achieving the maximum network lifetime. This cross-layer designated approach [1] was tested by simulation (NS2 simulator) and its performance over AODV [11], DSDV [12] was found to be better.
\end{abstract}

\section{General Terms}

Mobile Adhoc Networks (MANET), Cross Layer Framework, AODV and DSDV.

\section{Keywords}

Cross Layer based AODV (CLAODV), Ad hoc on Demand Distance Vector (AODV), Destination Sequenced Distance Vector Routing (DSDV).

\section{INTRODUCTION}

A mobile Ad hoc network (MANET) is a consistent, selfcomposing, infrastructure less network that is organized from claiming versatile apparatuses that need aid joined without wires. MANET [5] will be the new developing engineering which empowers clients will impart without any physical framework in any case for their geographic location, that's the reason it is frequently alluded on concerning an illustration of infrastructure less system.

Ad hoc networking permits the devices to deal with associations to maintain network connections and in addition effectively including and excluding device to and from the network. Because of nodal transportability, the system topology might transform quickly furthermore change unpredictably over time.

A critical issue prompting a constraint for mobile devices may be that they have this high mobility, causing connections to easily broken and then reestablished. Also, the bandwidth of remote wireless channel may be likewise limited, furthermore devices worked for set battery power, which will eventually be exhausted. Therefore, the mobile ad hoc network outline will be exceedingly challenging, but this technology need some way or another have figured out how to beat such limits with a percentage degree.

Previously, the design offered by ad hoc network protocol has mostly been based on the "layered approach". In layered architecture, the designer of the protocol algorithm focuses on a particular layer, without being required to consider the parameters of the rest of the stack. To overcome this, the "cross-layer [4]" approach has been found to address power consumption and network lifetime-related issues in wireless ad hoc networks.

The cross-layer design [1] deviates from the traditional design approach of network in which each layer of the stack would be made to operate independently. The inter-layer communication metrics and the benefits of information exchange among the lower layers such as network layer and transport layer were also reported.

In this paper, the simulation of Cross Layer based MANET framework to minimize energy consumption [3] and maximize network lifetime with comparison to Layered based MANET is proposed. Further, the different performance metrics such as PDR, energy and number of collisions based on simulated scenarios for Layered and Cross Layered based MANET are evaluated.

The remainder of this paper is classified as follows. The Related Research Work is presented in Section 2. Proposed Cross layer based AODV (CLAODV) is presented in Section 3. Results and Discussions obtained from the proposed work are presented in Section 4. and Conclusion of this paper is presented in Section 5.

\section{RELATED RESEARCH WORK}

A. Sarfaraz Ahmed, T. Senthil Kumaran , Mr. S. Syed Abdul Syed and S. Subburam [1] proposed a design approach, switching from the traditional layered architecture that has been found inefficient to cope with receiving signal strength 
(RSS)-related and power control problems which affects the physical layer, the network layer and the transport layer towards enhancing the cross-layer interaction among different layers.

M. Kanthirekha and Dr C.Satyanarayana [3] evaluated three ad hoc networks routing protocol such as EPAR, DSR and MTPR in different network parameter taking into consideration the power consumption. Certainly the proposed approach simulation of EPAR protocol while achieving QoS metrics like PDR, Throughput, Jitter, end-to-end Delay etc. along with the optimization of energy consumption.

M. Pratik Zala, I. Rajput and V. Gupta [4] have surveyed the different approaches of routing protocol that provide the stable path from source to destination will consume less energy and maximize the network life time.

P. Goyal, V. Parmar, and R. Rishi [5] described the fundamental problems occurred in ad hoc network by giving its related research background which actually deals with the concept, features, status, and vulnerabilities of MANET. It also includes the several challenging issues, emerging application and the future trends of MANET

I. Woungang, S. Kumar Dhurandher, S. Mohammad Obaidat and A. Ferworn, Waqas Shah [7] presented an AntColony Optimization (ACO) inspired approach to EEAODR. The obtained simulation results show that the ACO-EEAODR outperforms the EEAODR scheme in terms of energy consumed and overall network lifetime performance metrics.

P Seungjin, Y. Seong-Moo [8] have proposed a MAC protocol that not only guarantees reliable one-hop broadcast but also achieves it proficiently by exploring as many simultaneous executions of the communication as possible. In addition to the transmitted packets, the proposed algorithm utilizes the control packets that prevent packet collisions, and at the same moment, make the simultaneous communications possible to improve the network throughput.

A. Mesleh, N. Al-Zabin,Jihad Abdel-Jalil, H. Abu-Asbeh, N. Al-Mutlaq, H. Abu-Matter, M. Al-Hunati [11] proposed two energy-aware routing algorithms: an energyaware ad hoc ondemand distance vector i.e. e-AODV and an energy-aware dynamic source routing (e-DSR), and compares their performance with the well-known AODV and DSR routing algorithms.

T. Senthilkumaran, V. Sankaranarayanan [9] have introduced a method for dynamic congestion detection and control routing (DCDR) in ad hoc networks based on the approximations of the average length of queue at the node level. Using the average queue length, a node detects the present level of congestion and sends a warning message to its neighbors.

\subsection{Destination-Sequenced Distance-Vector Routing (DSDV)}

The Destination Sequenced Distance Vector Routing which is abbreviated as DSDV [12] is a table-driven routing approach for ad hoc mobile networks based on the traditional BellmanFord algorithm. It eliminates the route looping, increases convergence speed, and reduces overhead of control message.

In DSDV [12], each node maintains a next-hop table, where it exchanges this information with its corresponding neighbors. The two types of next-hop table exchange are: full-table broadcast and event-driven updating. The relative frequency of the full-table broadcast and the cumulative updating is determined by the node mobility. In each data packet sent during a next-hop table broadcast, the source node appends a sequence number. This sequence number is propagated by all nodes receiving the approaching distance-vector updates, and is stored in the next-hop table entry of nodes. The node, after receiving the current next-hop table from its neighbor, updates its route to a destination only if the current sequence number is greater than the recorded one, or if the new sequence number is found to be the same as the recorded one, but the new route obtained is shorter. In order to further reduce the overhead of control message, a settling time is estimated for each obtained route. A node updates its neighbors with a new route only if the settling time of the route has expired and the route remains optimal.

\subsection{Adhoc On Demand Distance Vector (AODV)}

It is known that AODV $[6,11]$ (Perkins \& Royer, 1999) is an improvement to the destination sequenced distance vector (DSDV) routing algorithm. To reduce the number of broadcast messages forwarded throughout MANETs, AODV [19] discovers routes on demand.

When a source node that seeks sending data packets to a destination node checks its route table for an optimum route to the destination node, if exists, it transmits the data packets to the next hop along the way to the destination. On the other hand, if a desired route does not exist in the route table of the source node, it starts a route discovery procedure by broadcasting a route request packet. to its immediate neighbors and if any intermediate neighbor with efficient enough energy, it adds the local energy of the intermediate node to the accumulated total energy field in the route request packet and it then broadcasts the route request packet further to its neighbors until the route request either reaches an intermediate node with a valid route to the destination or the destination node itself

\section{PROPOSED CROSS-LAYER BASED AODV (CLAODV)}

Energy proficient routing [17] over MANET [5] is a testing objective which needs to be made under thought. In particular, power effective routing may be a chance to be the majority vital outline criteria to MANETs since mobile nodes will be powered by the batteries with limited capacity. Power failure of a mobile node not only influence the node itself as well as its ability of transmitting packets and as a result affecting the overall network lifetime [2].

Institutionalization of layered protocol stacks has enabled quick improvement of interoperable systems, but at the same time long haul restricted the execution of the general architecture, because of the absence of coordination among layers which has failed to provide the efficiency in minimization of overall energy consumption and therefore, maximizing the network lifetime. Cross-layer Framework [4, 16] is an escape from the layered architecture of the OSI communications model with virtually strict boundaries between layers. The proposed Cross layer based AODV (CLAODV) removes such strict boundaries which opposes the communication between the layers that is to allow one layer to access the data of another layer so that the information exchange and interaction is enabled. This Cross Layer based MANET framework is simulated with the help of Network Simulator (NS2) [20] to minimize the power consumption and maximize the network lifetime with comparison to MANET.The desired information is passed 
from the physical layer to the network layer so that it can take optimum decisions required in the routing protocols. A major advantage provided by this approach is to allow access of information between physical layer and top layers such as MAC and network layer. The illustration of the cross-layer interaction among the layers is given in Figure 1.

\section{RESULTS AND DISSCUSSION}

The simulation results are obtained by the following parameters used in the scenario which is presented in Table 1. The simulation network consists of 50 nodes randomly scattered in a $1000 \times 1000 \mathrm{~m}$ area. At the beginning of the simulation, nodes are moving with a speed range between 0 to $35 \mathrm{~m} / \mathrm{s}$.

\subsection{Performance Metrics}

Performance of the simulated algorithms is evaluated in terms of packet delivery ratio, energy consumed and number of collisions occurred are defined as follows:

Packet delivery ratio (PDR): The ratio of number of packets that are successfully received by destination to the total number of packets sent by source is defined as the packet delivery ratio. This metric is expressed in percentage. Mathematically it is calculated by the following equation:

$\mathrm{PDR}=$ Number of received packets/Number of packets sent

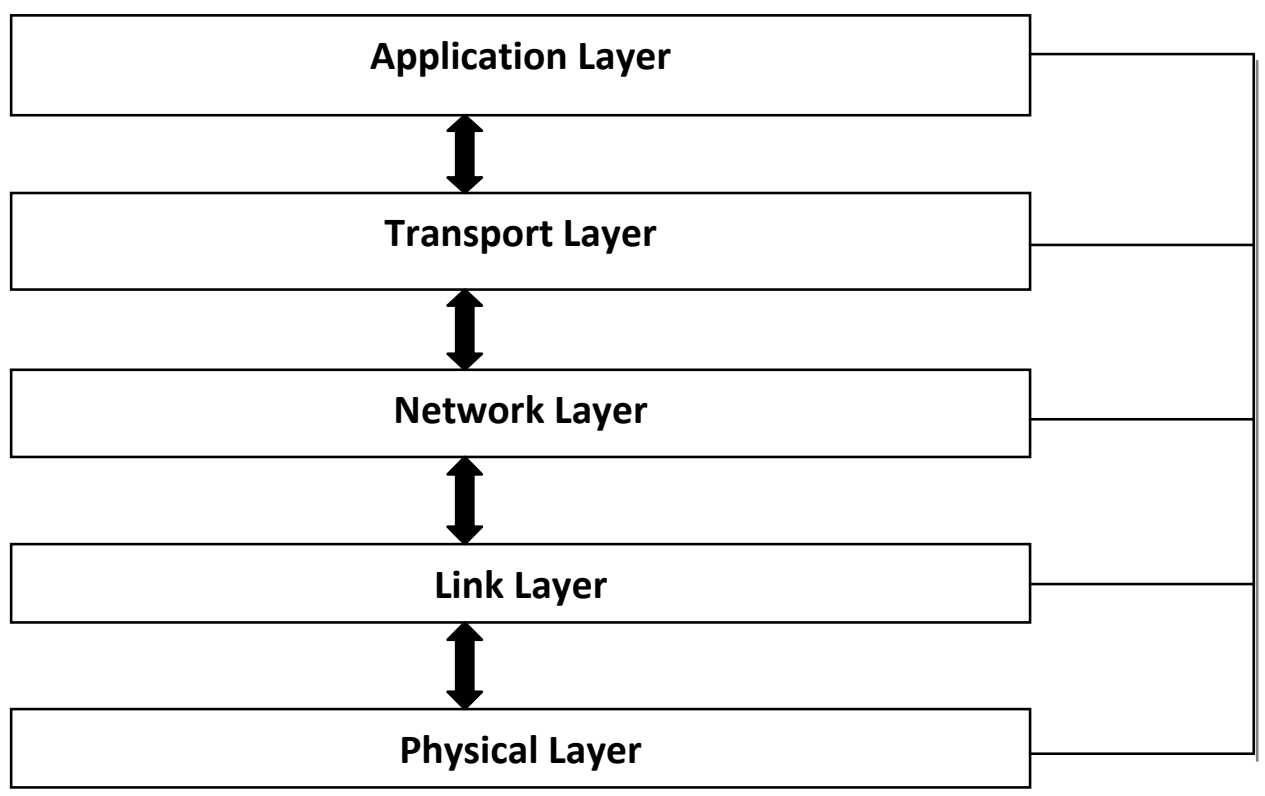

Fig 1: Cross Layer Interactions among the layers

Energy Consumed: The energy consumption of a node at any time of the simulation can be determined by finding the difference between the current energy value and Initial

Energy Consumed= (Initial Energy value-Current Energy value)/Number of nodes

Number of Collisions: In a network, when two or more nodes attempt to transmit a packet across the network at the same time, a packet collision occurs causing the packets to drop.

Number of Collisions $=$

Number of packets sent-Number of packets received Total simulation time

\subsection{Varying the node velocity}

In Figure 2, the comparison of three such as DSDV, AODV and CLAODV showing the variation of packet delivery ratio with respect to the increasing velocity of nodes in the network. We observe the impact of node mobility (i.e., node velocity) and offered network load on packet delivery ratio (PDR). In general, it is noticed that the PDR decreases as the node speed increases. PDR is $5 \%$ to $15 \%$ better with CLAODV, for higher node speeds (i.e., > $10 \mathrm{~m} / \mathrm{s}$ ), as compared to DSDV and AODV. However, for low mobility (i.e., node speed $\leq 5 \mathrm{~m} / \mathrm{s}$ ), DSDV, AODV, shows little variation when compared to proposed CLAODV. This is due to the network topography does not change significantly when the nodes are less mobile.

Table: 1 Simulation Parameters

\begin{tabular}{|c|c|}
\hline Parameter & Value \\
\hline No. of Nodes & 50 \\
\hline Terrain Area & $1000 \times 1000 \mathrm{~m}$ \\
\hline MAC Protocol & 802.11 \\
\hline Routing Protocol & AODV,DSDV,CLAODV \\
\hline Packet Size & 512 Byte \\
\hline Propagation Range & $200 \mathrm{~m}$ \\
\hline Traffic type & CBR \\
\hline Node Speed & $0-35 \mathrm{~m} / \mathrm{s}$ \\
\hline
\end{tabular}




\begin{tabular}{|c|c|}
\hline Simulation Time & $900 \mathrm{~s}$ \\
\hline Propagation Model & Two Ray Ground Model \\
\hline Data Rate & $1 \mathrm{Mbps}$ \\
\hline
\end{tabular}

In Figure 3 the graph shows the variation of energy consumed in DSDV, AODV and CLAODV with respect to the increasing node velocity from 0 to $35 \mathrm{~m} / \mathrm{s}$. When the node velocity is lower $(0-10 \mathrm{~m} / \mathrm{s})$ the energy consumed by CLAODV is reduced to $10 \%$ over AODV and $8.7 \%$ over DSDV and when the node velocity is higher $(15-35 \mathrm{~m} / \mathrm{s})$ the energy consumed by CLAODV is reduced by $17.7 \%$ over AODV and $24 \%$ over DSDV.

Figure 4 shows the performance metric Number of collisions w. $r$. $t$ the varying node velocity for the simulation scenario with various routing protocols such as DSDV, AODV and CLAODV. The variation of number of collisions that occurred with respect to the increasing velocity of node form 0 to $35 \mathrm{~m} / \mathrm{s}$ is shown. When the velocity of node is lower ( 0 to $10 \mathrm{~m} / \mathrm{s}$ ) the number of collisions in CLAODV reduced by $9 \%$ from AODV and 20\% in DSDV whereas when the node velocity was higher $(20-35 \mathrm{~m} / \mathrm{s})$ the number collisions in CLAODV reduced to $26.9 \%$ from AODV and $37 \%$ in DSDV.

\section{CONCLUSIONS}

The effective power consumption is a testing objective which needs to be taken under consideration. Previously, power effective routing may be the majority imperative outline criteria for MANETs since mobile nodes will be powered by batteries with restrained capacity. Power failure of a mobile node not only affect the node itself but also its ability to forward packets as a result decreasing the network lifetime.

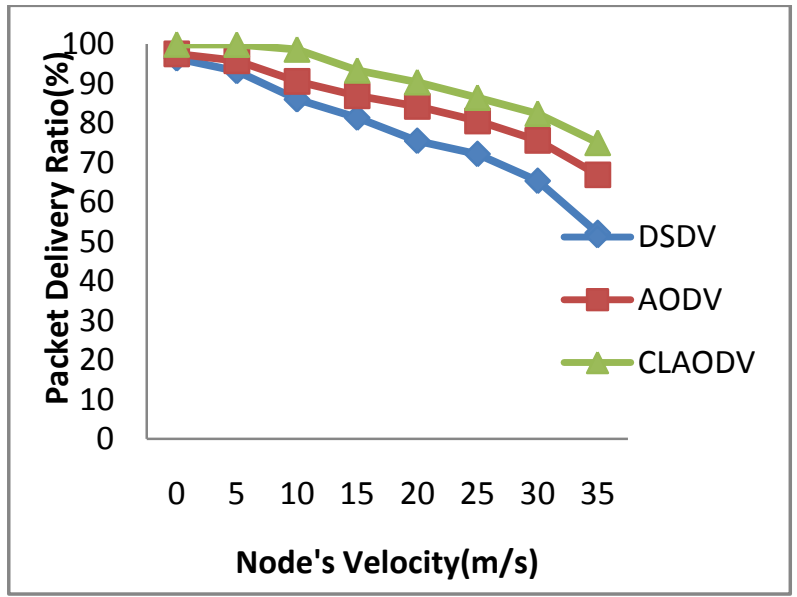

Figure 2 Packet Delivery Ratio Vs Node Velocity $(\mathrm{m} / \mathrm{s})$

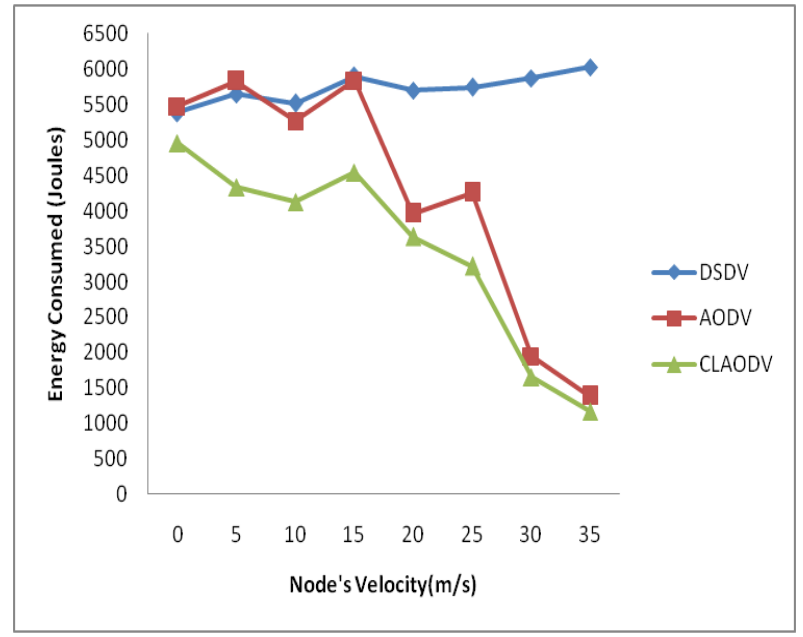

Figure 3 Energy Consumed (Joules) Vs Node Velocity $(\mathrm{m} / \mathbf{s})$

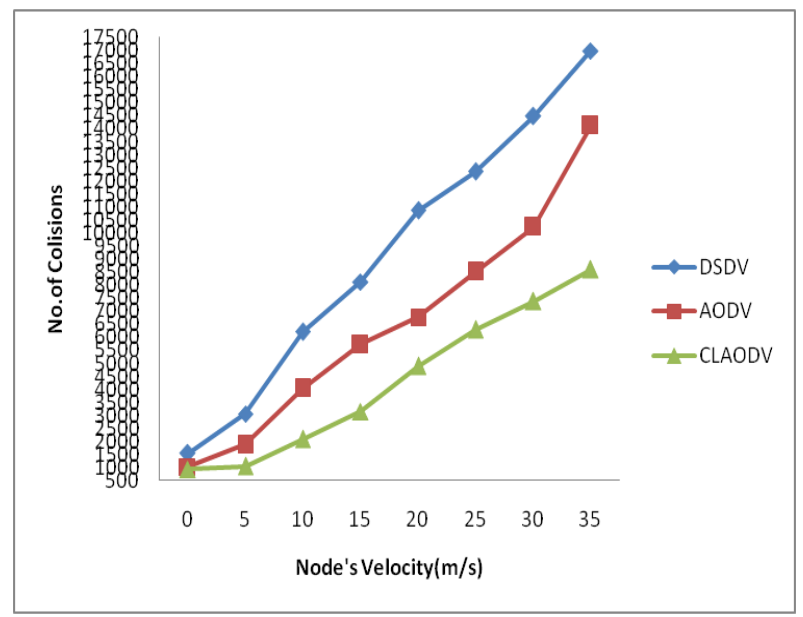

Figure 4 Number of collisions Vs Node Velocity $(\mathrm{m} / \mathrm{s})$

In our dissertation we have simulated a Cross Layer based MANET framework to minimize the power consumption [17] and maximize the network lifetime with comparison to MANET. We have performed the comparative analysis of the routing protocols such as DSDV, AODV with the proposed model that is Cross Layer based AODV (CLAODV). By simulation, the performance of CLAODV was appreciably better than DSDV and AODV in terms of packet delivery ratio, end-to-end delay, throughput, energy consumed.

\section{ACKNOWLEDGMENTS}

The author express her genuine much appreciated thanks to the expert Er. Pooja Saini, Sr. Assistant Professor, Ambala College of Engineering and Applied Research, Mithapur, Ambala, Haryana who have contributed towards development of my work.

\section{REFERENCES}

[1] Sarfaraz Ahmed , T. Senthil Kumaran , S. Syed Abdul Syed and S. Subburam "Cross-Layer Design Approach for Power Control in Mobile Ad Hoc Networks" in Egyptian Informatics Journal (ELSEVIER) Vol.no.16, December 2014, pp. 1-7.

[2] Shivshankar, Hosahalli, N. Suresh, G. Varaprasad, and G. Jayanthi "Designing Energy Routing Protocol With Power Consumption Optimization in MANETs" in 
Transaction in Emerging Topics of Computing (IEEE), Vol.no.2(2), July 2014, pp. 192-197.

[3] M. Kanthirekha and Dr C.Satyanarayana "Implementing Energy Routing Protocol for Optimization of Power Consumption in MANET" in International Journal of Advanced Research in Computer Science and Software Engineering (IJARCSSE), Vol.no.5, September 2015, pp. 341-345.

[4] M. Pratik Zala, I. Rajput and V. Gupta "Cross Layer Optimization Based On Energy Aware Routing Protocol For Manet" in International Journal For Technological Research In Engineering (IJTRE), Vol.no.1, June 2014, pp. $1165-1168$

[5] P. Goyal, V. Parmar, and R. Rishi, "MANET Vulnerabilities, challenges, attacks, application" in M International Journal of Computational Engineering \& Management(IJCEM), Vol.no.11, January 2011, pp. 3237.

[6] R Gowada and S. Ramanna Havinal "Cross Layer Energy Improvement in MANET" in International Journal of Engineering Science and Innovative Technology (IJESIT), Vol.no.2, March 2013, pp. 72-82.

[7] S. Mishra, S. Mohan Satpathy and A. Mishra "ENERGY EFFICIENCY IN AD HOC NETWORKS" in International Journal of Ad hoc, Sensor \& Ubiquitous Computing (IJASUC) Vol.no.2(1), March 2011, pp. 1-5.

[8] P. Seungjin and Y. Seong-Moo "An efficient reliable one-hop broadcast in mobile ad hoc networks" in Journal in adhoc networks (ELSEVIER), Vol. no.11 (1) 2013, pp. 19-28.

[9] T. Senthilkumaran, V. Sankaranarayanan "Dynamic congestion detection and control routing in ad hoc networks" in Journal of King Saud University 'Computer and Information Sciences (Elsevier), Vol.no.25, May 2012, pp. 25-34

[10] A. Mesleh, N. Al-Zabin,Jihad Abdel-Jalil, H. AbuAsbeh, N. Al-Mutlaq, H. Abu-Matter, M. Al-Hunati "AODV and DSR energy-aware routing algorithms: a comparative study" in International Journal of Computer Science (IJCSI), Vol.no.9, November 2012, pp. 266-271.

[11] N. Kumar, Dr. C. Suresh Gnana Dhass "A Complete Study on Energy Efficient Routing Protocols DSR, ZRP and DSDV In Mobile Ad Hoc Networks" in The International Journal Of Engineering And Science (IJES), Vol. no. 1, July 2012, pp. 54-60.
[12] T. Preet Singh, S. Dua and V. Das "Energy-Efficient Routing Protocols In Mobile Ad-Hoc Networks" in International Journal of Advanced Research in Computer Science and Software Engineering (IJARCSSE), Vol.no.2, January 2012, pp. 24-31.

[13] C. Poongodi and A. M. Natarajan, "Optimized replication strategy for intermittently connected mobile networks" in International Journal of Business Data Communication Network (IRMA), Vol.no.8(1), January 2012, pp. 1-3.

[14] T.C. Huang, S.C. Chen and L.Tang "Energy-Aware Gossip Routing for Mobile Ad Hoc Networks"in International Conference on High Performance Computing and Communications (IEEE), Vol.no.2, September 2011, pp. 955-959.

[15] T. Senthilkumaran and V. Sankaranarayanan "Early congestion detection and adaptive routing in MANET" in Egyptian Informative Journals (ELSEVIER), Vol.no.12, October 2011, pp. 165-75.

[16] S. Sergi, F. Pancaldi and G.M. Vitetta "Cross-layer design for double string cooperative communications in wireless ad-hoc networks" in European Transaction of Telecommunication, (WILEY), Vol.no.22, December 2011, pp. 471-486.

[17] A.Rajaram and J. Sugesh, "Power aware routing for MANET using on-demand multipath routing protocol" in International Journal of Computer Science (IJCSI), Vol.no.8, July 2011, pp. 517-522.

[18] Shah, H. Gupta and M. Baghel "Energy efficient routing protocols for mobile ad hoc networks" in International Journal of Engineering Research and Technology (IJERT), Vol.no.1, July 2012, pp. 1- 5.

[19] P. Nayak, R. Agrawal and S. Verma "Energy aware routing scheme for mobile ad hoc network using variable range transmission" in International Journal of Ad hoc Sensor and Ubiquitous Computing (IJASUC), Vol.no.3, July 2012, pp. 53-63.

[20] Mrs. Saba Siraj, Mr. Ajay Kumar Gupta , Mrs RinkuBadgujar, "Network Simulation Tools Survey" in International Journal of Advanced Research in Computer and Communication Engineering, Vol.no.1, June 2012, pp. 201-210.Tavel, P. 2007 Modeling and Simulation Design. AK Peters Ltd. 